Bull. Mater. Sci., Vol. 22, No. 3, May 1999, pp. 531-535. (C Indian Academy of Sciences.

\title{
Quantum dots fabricated by selective area MOVPE and their application to single electron devices
}

\author{
TAKASHI FUKUI*, F NAKAJIMA, K KUMAKURA, and J MOTOHISA \\ Research Center for Interface Quantum Electronics, Hokkaido University, N13, W8, Sapporo 060-8628, Japan
}

\begin{abstract}
A novel method of formation of uniform GaAs quantum dot (QD) structures, using selective area metalorganic vapour phase epitaxy (SA-MOVPE), and their application to single electron transistors (SETs) are demonstrated. The $\mathrm{SiN}_{x}$-coated substrates having a wire-like opening with three prominences are used. The wire-like opening is aligned in the [110] direction, which corresponds to channel region of SET. AlGaAs/GaAs modulation-doped heterostructures are grown on these substrates. Due to three prominences on the wire, the quasi-one-dimensional electron gas (Q-1DEG) channel, having a periodic variation in its width, are naturally formed. This leads to the formation of a quantum dot near the central prominence and two tunneling barriers beside the dot, which are connected to quantum wires. $I_{\mathrm{D}}-V_{\mathrm{G}}$ characteristics under constant source-drain bias condition show clear conductance oscillations near the pinch-off, and oscillations are observed up to $65 \mathrm{~K} . I_{\mathrm{D}}-V_{\mathrm{DS}}$ characteristics measured at $2 \cdot 1 \mathrm{~K}$ show clear Coulomb blockade. The results indicate the formation of SET by SA-MOVPE. Using similar method, resistance-load single electron inverter circuit is also fabricated.
\end{abstract}

Keywords. Quantum dots; MOVPE; single electron devices.

\section{Introduction}

Semiconductor quantum wires (QWRs) and quantum dots (QDs) have attracted much attention for new optical device applications, such as quantum dot lasers. Many researchers have reported $\mathrm{GaAs}$ and $\operatorname{In}(\mathrm{Ga}) \mathrm{As}$ QD structures fabricated using selective area metalorganic vapour phase epitaxy (SA-MOVPE) (Fukui et al 1991; Nagamune et al 1993; Lebens et al 1990; Kumakura et al 1997), and self-organized growth (Leonard et al 1993; Temmyo et al 1995; Bimberg et al 1996). High density In(Ga)As QDs were automatically formed on GaAs substrate by the strain effects, and QD lasers were reported using this self-organized growth mode (Temmyo et al 1995; Bimberg et al 1996).

At the same time, new quantum functional devices, which consist of such QWRs and QDs, have attracted equal attention, in particular, for fabrication of single electron transistors (SETs), and electron wave interference devices (EWIDs). It is important to connect these QWRs and QDs with tunneling barriers, so as to form the periodically-modulated potential energy.

SA-MOVPE is one of the useful techniques for fabrication of these quantum nanostructures (Fukui et al 1991), and has been applied to fabricate optical integrated circuit (Aoki et al 1991). Using this selective growth, uniform QD structures can be formed by adjusting the growth conditions as well as substrate orientation and mask pattern, for example GaAs (001) (Nagamune et al

*Author for correspondence
1993; Lebens et al 1990; Kumakura et al 1995), and GaAs (111)B (Fukui et al 1991) substrates. QD-network, which is composed of QDs connected with QWRs, was also reported in our previous study (Kumakura et al 1997). Thus, SA-MOVPE method holds a promise for its future use in optical devices and optoelectronic integrated circuits (OEIC). The application of QDs to electron transport devices, especially single electron devices, is also a very important research field for future electron devices. For fabrication of these devices, these well-arranged QDs have to be connected to each other. To realize the SET structure, we attempted to fabricate quantum dot-tunnel barrier systems by molecular beam epitaxy on patterned substrates (Dilger et al 1996).

In this paper we have presented fabrication process of GaAs SETs using GaAs/AlGaAs modulation-doped structures grown on the masked substrates by SAMOVPE, and clarify the transport properties. SETs showed Coulomb blockade type conductance oscillation near the pinch-off voltage up to $65 \mathrm{~K}$. Coulomb gap and total capacitance $C_{\Sigma}$ was estimated to be $12 \mathrm{mV}$ and $13 \mathrm{aF}$, respectively.

\section{Fabrication process and device structure}

Selective area (SA-) MOVPE growth was carried out using a low-pressure horizontal, RF-heated, quartz reactor system. The working pressure of 76 Torr was automatically controlled. Purified hydrogen $\left(\mathrm{H}_{2}\right)$ was used as a carrier gas. The source materials were trimethylgallium 
(TMGa), trimethylaluminum (TMAl) and $20 \%$ arsine $\left(\mathrm{AsH}_{3}\right)$ in $\mathrm{H}_{2}$. The partial pressures of TMGa and TMAl were kept constant at $3.8 \times 10^{-6}$ and $6.3 \times 10^{-7} \mathrm{~atm}$, respectively. The partial pressure of $\mathrm{AsH}_{3}$ was $1.3 \times 10^{-4} \mathrm{~atm}$ for GaAs buffer layer, and $6.7 \times 10^{-4} \mathrm{~atm}$ for $\mathrm{Al}_{0.3} \mathrm{Ga}_{0.7}$ As layer to achieve better quality, respectively. Growth rates of $\mathrm{GaAs}$ and $\mathrm{Al}_{0.3} \mathrm{Ga}_{0.7}$ As were 0.93 and $1.5 \mu \mathrm{m} / \mathrm{h}$ for planar substrates, respectively.

SA-MOVPE was carried out on the masked substrates at the growth temperature of $700^{\circ} \mathrm{C}$. The layer sequences are as follows; a $400 \mathrm{~nm}$ GaAs buffer layer, a $50 \mathrm{~nm}$ $\mathrm{Al}_{0.3} \mathrm{Ga}_{0.7} \mathrm{As}$ layer, a $15 \mathrm{~nm}$ GaAs well layer, a $10 \mathrm{~nm}$ undoped $\mathrm{Al}_{0.3} \mathrm{Ga}_{0.7} \mathrm{As}$ layer, a $50 \mathrm{~nm} n$-doped $\mathrm{Al}_{0.3} \mathrm{Ga}_{0.7} \mathrm{As}$ layer, and a $10 \mathrm{~nm} n$-doped GaAs capping layer. The mobility and sheet electron concentration of two-dimensional electron gas (2DEG) grown on a planar substrate were $62,000 \mathrm{~cm}^{2} / \mathrm{V}$.s and $1.2 \times 10^{12} \mathrm{~cm}^{-2}$ at $77 \mathrm{~K}$, respectively.

Schematic illustration of the mask pattern for SET structure is shown in figure 1a. The pattern is formed on semi-insulating (001) GaAs coated with $40 \mathrm{~nm}$-thick $\mathrm{SiN}_{x}$ layer by electron beam lithography and wet chemical etching. The wire-like opening with three prominences aligned in the [110] direction corresponds to channel region of SET.

Figure 1b shows SEM image after the selective growth, and figure $1 \mathrm{c}$ the schematic illustration of the device structure. The wire region consists of $\{111\} \mathrm{B}$ facet sidewalls, and $\{110\}$ facet sidewalls are formed near the prominences. A (001) plane appeared on the top portion of the whole structure. Due to three prominences on the wire, the quasi-one-dimensional electron gas (Q-1DEG) channel has periodic variation in its width and thickness. This leads to the formation of a quantum dot near the central prominence and two tunneling barriers beside a dot, which are connected to quantum wires. For reference, quantum wire was also grown on masked substrate without prominences. The channel width of the present Q-1DEG is $400 \mathrm{~nm}$ by SEM observation.

After the growth of these structures, source and drain $\mathrm{Ge} / \mathrm{Au} / \mathrm{Ni}$ ohmic electrodes were formed on the wide 2DEG regions (figure $1 \mathrm{~b}$ ) by a lift-off process followed by alloying at $350^{\circ} \mathrm{C}$ for $5 \mathrm{~min}$ in $\mathrm{N}_{2}$ atmosphere. Next, Schottky gate of $\mathrm{Al}$ electrode was formed by a lift-off process on the three prominences region. Since no growth occurs on $\{111\} \mathrm{B}$ facets, both edges of the Q-1DEG channel are exposed to air. Therefore, Schottky gate has direct contact at the edges of the Q-1DEG, and controls the electric field perpendicular to the edges. The gate length is $5 \mu \mathrm{m}$.

\section{Transport properties of GaAs single electron transistor structure}

Figure 2 shows $I_{\mathrm{D}}-V_{\mathrm{G}}$ characteristics of quantum dot structure connected with quantum wires with constant source-drain bias condition $\left(V_{D S}=200 \mu \mathrm{V}\right)$ for various temperatures. Clear conductance oscillations were observed near the pinch-off, and oscillations were observed up to $65 \mathrm{~K}$. We have already fabricated several devices with the same structure. Although the pinch-off gate voltages were slightly different in each device due to run-to-run fluctuation of the doping carrier density for the MOVPE growth, clear conductance oscillations were observed for these devices. These results suggest that three prominences in the channel region play an important role for the conductance oscillations.

Figure 3 shows typical $I_{D}-V_{D S}$ characteristics measured at $2.1 \mathrm{~K}$, which shows clear Coulomb blockade effect. Coulomb gaps and their modulation by the gate voltage were seen near the pinch-off voltage. The maximum
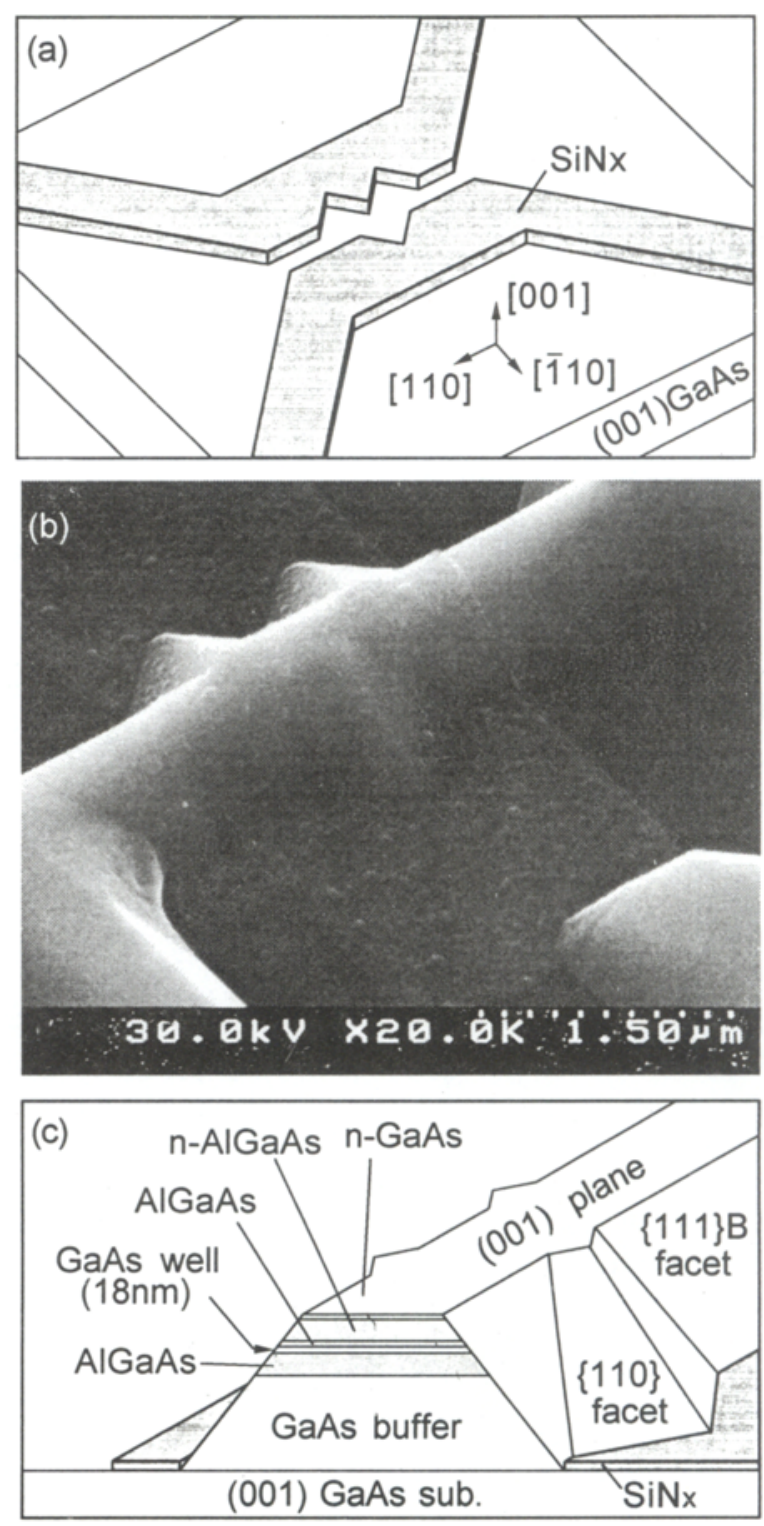

Figure 1. (a) Schematic illustration of $\mathrm{SiN}_{x}$ masked substrate for selective area MOVPE, (b) SEM image after the selective growth on the masked substrate, and (c) schematic illustration of the device structure. 
Coulomb gap $\Delta$ is about $12 \mathrm{mV}$, which corresponds to a thermal energy $k T$ of $140 \mathrm{~K}$. Following a simple model of Coulomb blockade, the total capacitance $C_{\Sigma}$ of the dot can be estimated to be about $13 \mathrm{aF}$ from $\Delta=e / C_{\Sigma}$. The radius $R$ of the dot is estimated to be about 14 $\mathrm{nm}$, using the relation of $C_{\Sigma}=8 \varepsilon \varepsilon_{0} R$.

The possible mechanism for the formation of small dot and tunneling barriers was as follows: When the depletion layer from the surface and both edges of Q-1DEG is expanded by applying the negative gate voltage, potential energy modulation was formed due to variation of effective channel width. The conductance oscillation far from the pinch-off voltage was thought to be the result of electron wave interference, similar to Si quantum dot (Leobandung et al 1995). Near the pinch-off voltage, the difference of the width of depletion layer from the edges of Q-1DEG and/or the thickness variation of $\mathrm{GaAs}$ channel due to three prominences may lead to formation of small dot and wires with tunneling barriers. Therefore, Coulomb blockade type conductance oscillation was observed near the pinch-off gate voltage.

\section{Resistance-load SET inverter circuit}

Next, we fabricated resistance-loaded SET inverter, which corresponds to that proposed by Likharev (1987). Figures $4 \mathrm{a}$ and $\mathrm{b}$ show a scanning electron microscope

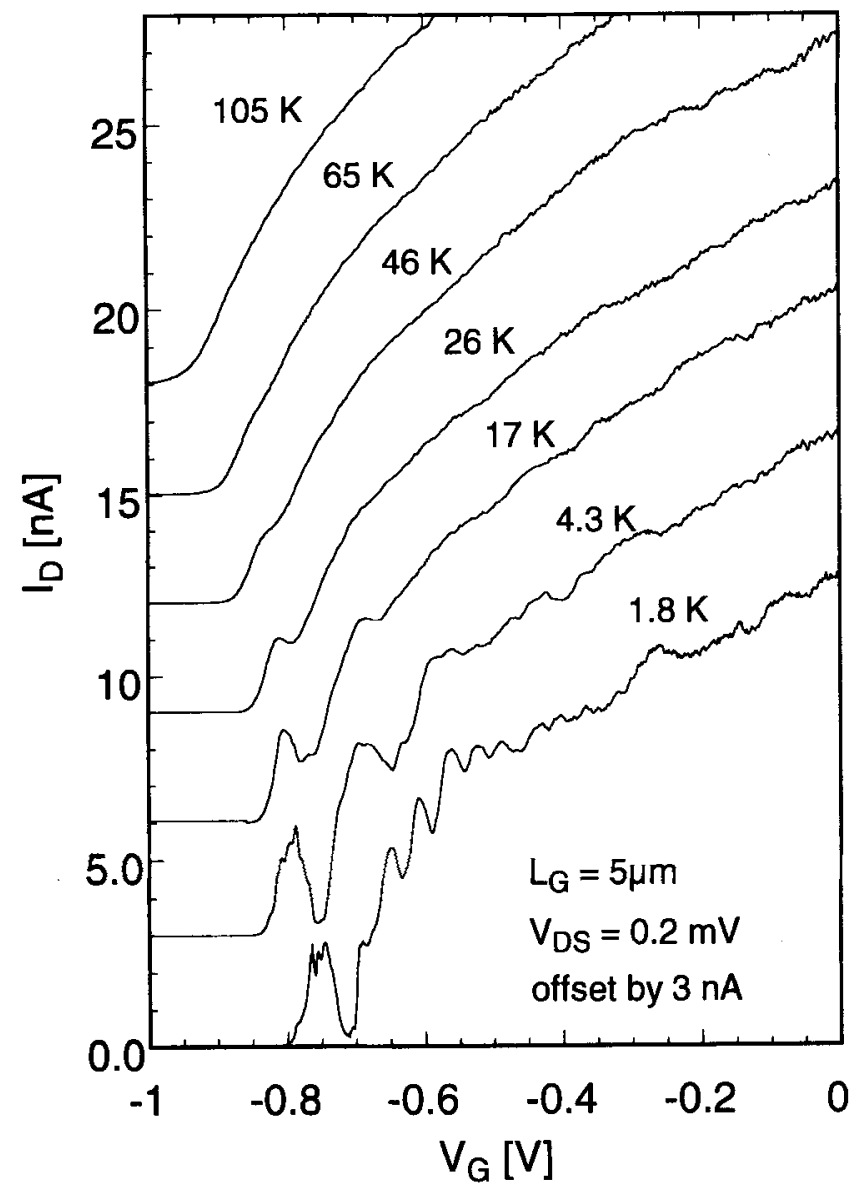

Figure 2. Temperature dependence of $I_{\mathrm{D}}-V_{\mathrm{G}}$ characteristics.

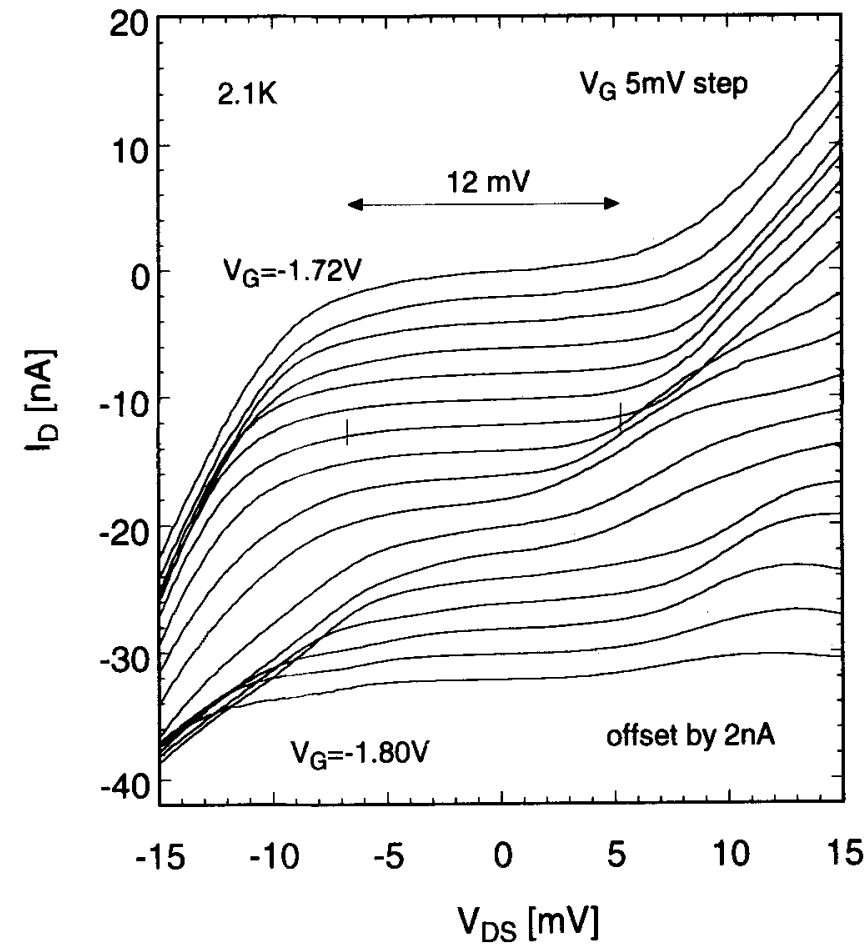

Figure 3. Typical $I_{\mathrm{D}}-V_{\mathrm{G}}$ characteristic at $2 \cdot 1 \mathrm{~K}$.

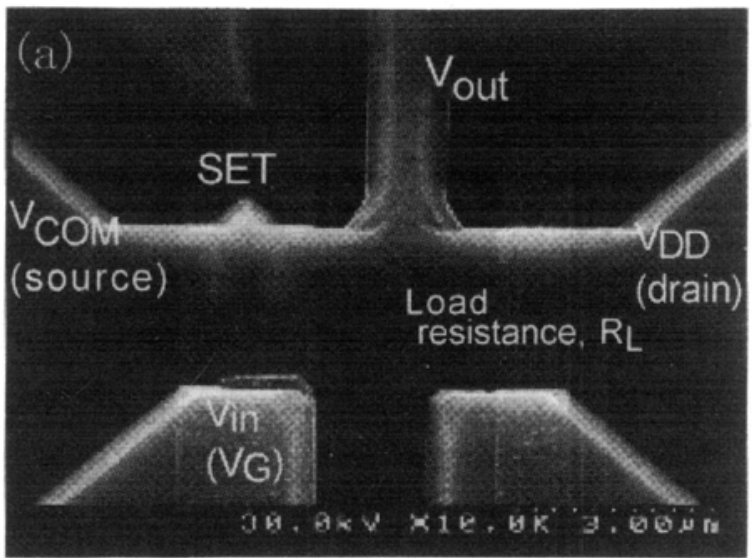

(b)

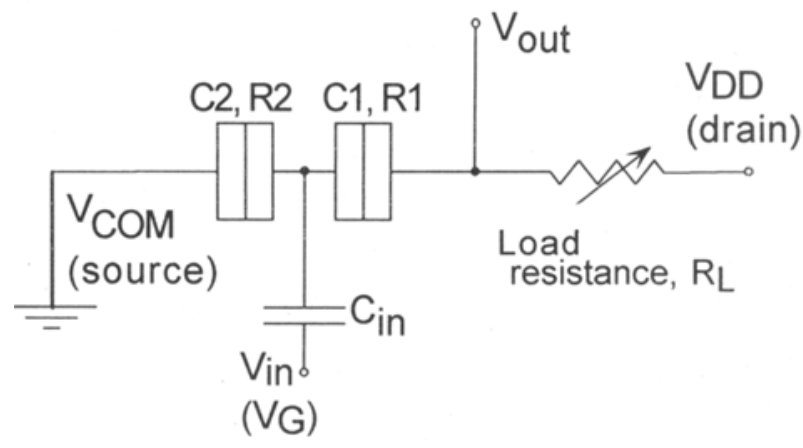

Figure 4. (a) SEM image of the device structure grown on this masked substrate, and (b) the equivalent circuit of the device, which corresponds to resistance-loaded SET inverter circuit proposed by Likharev (1987). 
(SEM) image of the device structure grown on the masked substrate and equivalent circuit of the device. In this device, Al Schottky contact was additionally formed on load resistance part. The length between source and drain electrodes was $10 \mu \mathrm{m}$, and the widths of Al Schottky contact for the SET- and $R_{\mathrm{L}}$-control gates were $2 \mu \mathrm{m}$ and $1 \mu \mathrm{m}$, respectively. It can be possible to set the optimized value of the $R_{\mathrm{L}}$ in the inverter circuit by only applying gate voltage, $V_{\mathrm{R}}$. Initial resistance value of $R_{\mathrm{L}}$ without applying the $V_{\mathrm{R}}$ was roughly estimated to be several $\mathrm{k} \Omega$, then, this value can be controlled, e.g. about $60 \mathrm{k} \Omega$ by applying the $V_{\mathrm{R}}$ of $-3.6 \mathrm{~V}$ in this experiment.

(a)

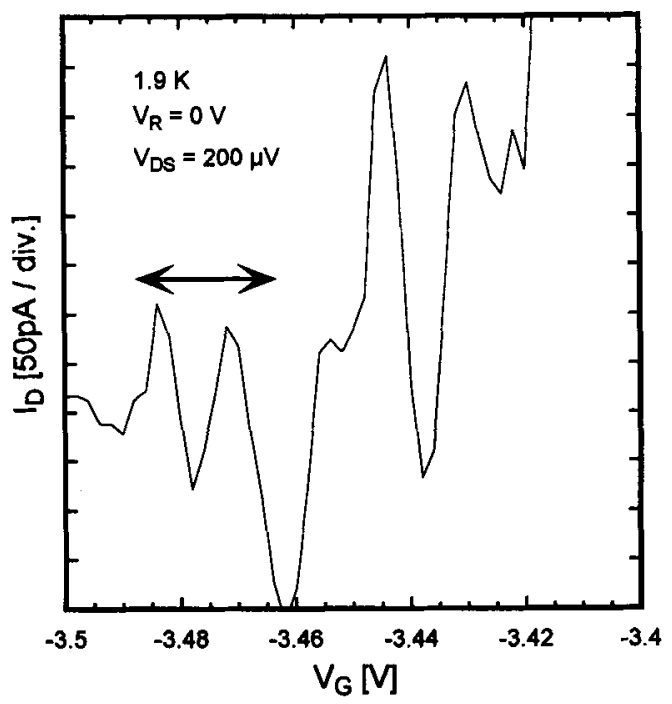

(b)

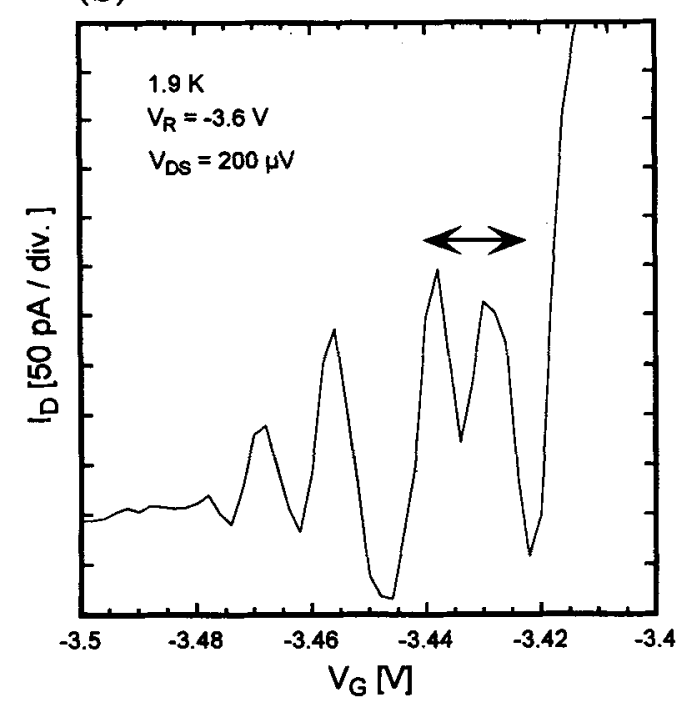

Figure 5. Drain current, $I_{\mathrm{D}}$ vs SET-control gate voltage, $V_{\mathrm{G}}$ characteristics of the device with $V_{R}$ of (a) $0 \mathrm{~V}$, and (b) $-3.6 \mathrm{~V}$ for the constant source-drain voltage, $V_{D S}$ of $200 \mu \mathrm{V}$ measured at $1.9 \mathrm{~K}$.
The drain current, $l_{\mathrm{D}}$ versus SET-control gate voltage, $V_{\mathrm{G}}$ characteristics of this device were measured at $1.9 \mathrm{~K}$ with $V_{\mathrm{R}}$ of $0 \mathrm{~V}$ and $-3.6 \mathrm{~V}$ for the constant sourcedrain voltage, $V_{D S}$ of $200 \mu \mathrm{V}$. Figures 5a and b show $I_{\mathrm{D}}-V_{\mathrm{G}}$ characteristics. Clear and similar conductance oscillations near the pinch off gate voltage were observed for both $V_{\mathrm{R}}$ of $0 \mathrm{~V}$ and $-3.6 \mathrm{~V}$. In particular, the periods of oscillation peaks were the same for both measurements, thus, $V_{R}$ did not affect the conductance oscillations at all. Figures $6 \mathrm{a}$ and $\mathrm{b}$ show $I_{\mathrm{D}}-V_{\mathrm{DS}}$ characteristics of this device with $V_{R}$ of $0 \mathrm{~V}$ and $-3.6 \mathrm{~V}$, and with different $V_{\mathrm{C}}$ values indicated in figures $5 \mathrm{a}$ and $\mathrm{b}$ by arrows, respectively. Clear Coulomb gaps and their modulations

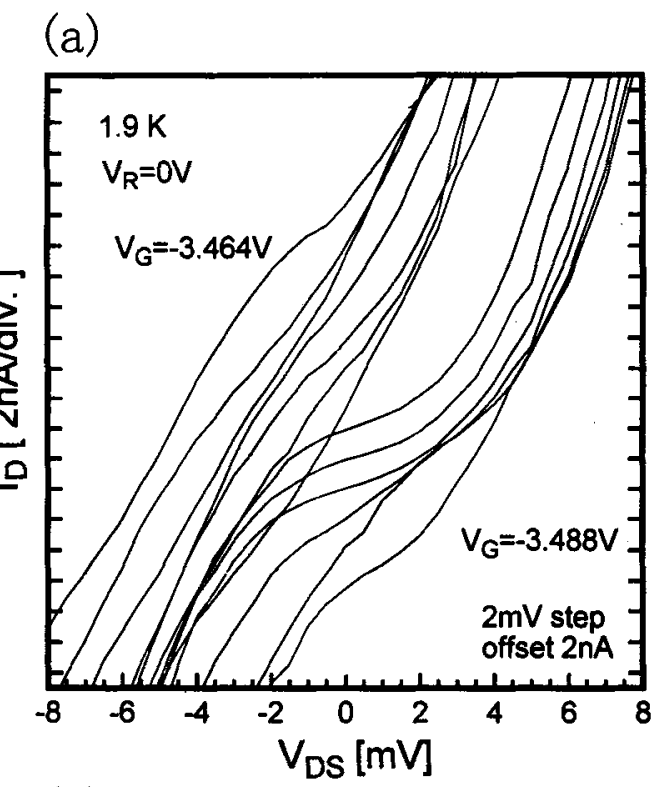

(b)

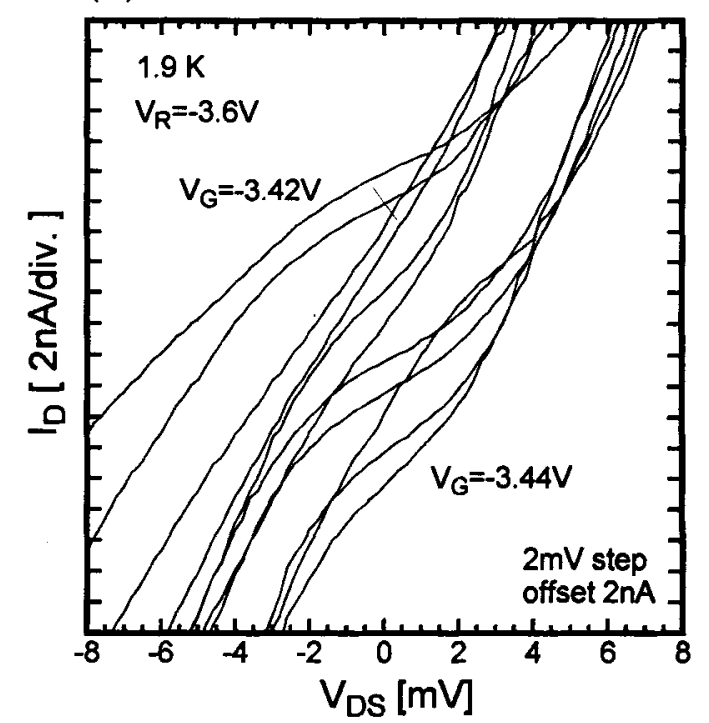

Figure 6. $I_{\mathrm{D}}-V_{\mathrm{DS}}$ characteristics of the device with $V_{\mathrm{R}}$ of (a) $0 \mathrm{~V}$, and (b) $-3.6 \mathrm{~V}$, and with different $V_{\mathrm{G}}$ values indicated in figures $5(\mathbf{a})$ and (b) by arrows. 


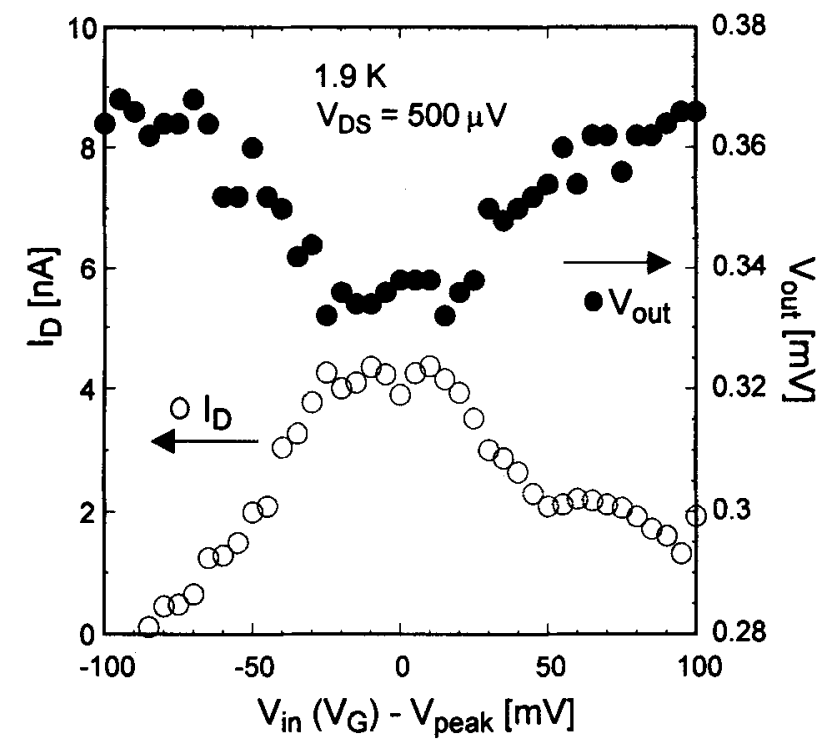

Figure 7. $V_{\text {out }}-V_{\text {in }}$ and $I_{\mathrm{D}}-V_{\text {in }}$ characteristics of this inverter circuit.

were observed for both cases. The maximum Coulomb gap in this sample is about $6 \mathrm{mV}$, and total capacitance, $C_{\Sigma}$ of the dot can be estimated to be about $27 \mathrm{aF}$, which corresponds to the dot radius of $28 \mathrm{~nm}$. These results indicate that $V_{\mathrm{G}}$ and $V_{\mathrm{R}}$ do not interfere with each other, and can be controlled independently.

Next, $I_{\mathrm{D}}-V_{\text {in }}$ (input voltage) and $V_{\text {out }}$ (output voltage) $-V_{\text {in }}$ characteristics were measured at $1.9 \mathrm{~K}$ under the constant $V_{\mathrm{DS}}\left(V_{\mathrm{dd}}\right)$ condition. Figure 7 shows $I_{\mathrm{D}}-V_{\text {in }}$ and $V_{\text {out }}-V_{\text {in }}$ characteristics of this inverter circuit. $V_{\mathrm{DS}}$ was $500 \mu \mathrm{V}$. $V_{\text {in }}$ corresponds to the gate voltage, $V_{\mathrm{G}}$ for the SET. Open and solid circles correspond to $I_{\mathrm{D}}$ versus $V_{\text {in }}$ and $V_{\text {out }}$ versus $V_{\text {in }}$ in the Coulomb blockade region near the pinch off gate voltage. $V_{\text {peak }}$ in the $x$-axis means one of the conductance peaks in the Coulomb oscillations. As $V_{\text {in }}$ was swept from $V_{\text {peak }}$ (i.e. $V_{\text {in }}-V_{\text {peak }}$ of zero) to the negative gate voltage, $V_{\text {out }}$ was smoothly increased, clearly showing the inverter operation. From $I_{D}-V_{G}$ and $I_{D}-V_{D S}$ measurements with applying the gate voltage, $V_{\mathrm{R}}$ to the $R_{\mathrm{L}}$, conductance oscillation was originated from Coulomb blockade effects. Although the value of the load resistance was set at $60 \mathrm{k} \Omega$ by applying the gate voltage, $V_{\mathrm{R}}, R_{\mathrm{L}}$ value was estimated to be about $7 \mathrm{k} \Omega$ from $I_{\mathrm{D}}$ and $V_{\text {vut }}$ characteristics. For $I_{\mathrm{D}}=0, V_{\text {out }}$ should be $0.5 \mathrm{mV}$, but actual value of $V_{\text {out }}$ was $0.37 \mathrm{mV}$. These discrepancies are not well understood yet. At present, the logic swing, $\Delta V_{\text {out }}$ relative to the $\Delta V_{\text {in }}\left(\Delta V_{\text {out }} / \Delta V_{\text {in }}\right)$ is small. We can fabricate higher gain inverter circuit for further optimi- zation of device parameters, such as, the value of load resistance, tunneling resistance, dot size and so on.

\section{Summary}

We have demonstrated GaAs quantum dot connected with GaAs quantum wires by SA-MOVPE, and applied these structures to GaAs SETs and resistance-load single electron inverter circuit. Their transport properties were investigated at low temperature. In SETs, near the pinchoff voltage, Coulomb blockade type conductance oscillations were observed up to $65 \mathrm{~K}$. Coulomb gap and total capacitance $C_{\Sigma}$ were estimated to be $12 \mathrm{mV}$ and $13 \mathrm{aF}$, respectively. Next, the inverter operation using this SET with load resistance was confirmed. Therefore, it is be possible to form the SET integrated circuits by further optimization of the mask shape, growth conditions and device parameters.

\section{Acknowledgements}

The authors wish to thank Prof. H Hasegawa for fruitful discussion and encouragement, and greatly acknowledge Dr S Hara, Y Oda, and T Umeda for technical assistance in MOVPE growth.

\section{References}

Aoki M, Sano H, Suzuki M, Takahashi M, Uomi K and Takai A 1991 Electron. Lett. 272138

Berggren K-F, Roos G and van Houten H 1988 Phys. Rev. B37 10118

Bimberg D et al 1996 Jpn J. Appl. Phys. 351311

Dilger M, Haug R J, Eberl K, Kurtenbach A, Kershaw Y and Klitzing K V 1996 Appl. Phys. Lett. 683132

Fukui T, Ando S, Tokura Y and Toriyama T 1991 Appl. Phys. Lett. 582018

Kumakura K, Nakakoshi K, Motohisa J, Fukui T and Hasegawa H 1995 Jpn J. Appl. Phys. 344387

Kumakura K, Motohisa J and Fukui T 1997 J. Crystal Growth 170700

Lebens A, Tsai C S, Vahala K J and Kuech T F 1990 Appl. Phys. Lett. 562642

Leobandung E, Guo L, Wang Y and Chou S Y 1995 Appl. Phys. Lett. 67938

Leonard D, Krishnamurthy M, Reaves C M, Denbaars S P and Petroff P M 1993 Appl. Phys. Lett. 633203

Likharev K K 1987 IEEE Trans. Magn. MAG-23 1142

Nagamune Y, Tsukamoto S, Nishioka M and Arakawa Y 1993 J. Crystal Growth 126707

Temmyo J, Kuramochi E, Sugo M, Nishiya T, Nötzel R and Tamamura T 1995 Electron. Lett. 31209 